\title{
Kommentar
}

\section{Wie viele Parkinson-Medikamente brauchen wir?}

\author{
Kürzlich wurde bekannt, dass Budipin Ende 2018 vom Markt genom- \\ men werden soll. Viele Ärzte kennen dieses Medikament kaum, auch \\ unter Neurologen hat die Mehrzahl es nur selten eingesetzt. Der Ver- \\ lust scheint also nicht so schlimm zu sein, die wenigen Patienten, die es \\ erhalten, und die wenigen Ärzte, die es verordnen, werden es schon \\ verkraften. Das entspricht wohl dem aktuellen Zeitgeist, ist meines \\ Erachtens aber sehr zu bedauern und macht unsere Therapie ärmer.
}

$\mathrm{B}$ udipin ist eine seit vielen Jahren bekannte Substanz, dessen Hauptindikation in der Behandlung des Tremors gesehen wird, darüber hinaus kann sie aber auch bei allen anderen motorischen Kernsymptomen in jedem Stadium der Parkinson-Erkrankung eingesetzt werden. Budipin wird meist bei Patienten mit tremordominantem Parkinson-Syndrom verordnet, wenn andere Dopaminergika nicht ausreichend helfen. Aufgrund der möglichen QT-Zeit-Verlängerung und eventueller Torsade-despointes-Tachykardien sind eine Aufklärung des Patienten sowie regelmäßige EKG-Kontrollen vorgeschrieben (vor Therapiebeginn, nach einer und drei Wochen und bei späteren Dosiserhöhungen Bestimmung der QTc [ $<420 \mathrm{~ms}$, QTcAnstieg $<60 \mathrm{~ms}]$ ). Deshalb erhalten nur wenige Patienten diese Substanz und das Produkt ist für den Hersteller defizitär, weshalb er den Vertrieb einstellt.

Zwar sind nur wenige Patienten auf Budipin eingestellt, es ist aber genau für diese ein großer Verlust, wenn Sie das Medikament nicht mehr erhalten können. Würde ihr Tremor nämlich auf andere Substanzen gut ansprechen, hätte man Budipin gar nicht erst versucht. Die Option Clozapin besteht zwar auch noch, es ist für diese Indikation aber nicht zugelassen und durch die Pflicht belastet, regelmäßig Blutbildkontrollen durchzuführen. Viele Patienten stehen dem Prä- parat daher zurückhaltend gegenüber und etliche Ärzte verordnen es nicht oder nur sehr ungern. Auch Anticholinergika reduzieren den Tremor, haben aber erhebliche unerwünschte Wirkungen, weshalb wir sie nur selten einsetzen. Die tiefe Hirnstimulation ist beim Tremor sehr gut wirksam, ein direkter Vergleich dieser aufwendigen, invasiven Methode mit einer oralen Medikation wäre aber nicht angemessen.

Akinese und Rigor sprechen gut auf Levodopa und andere dopaminerge Substanzen an, aber viele Patienten mit Tremor profitieren nur unzureichend, weshalb sie auf andere Ansätze angewiesen sind. Auch die anderen Symptome sowie die motorischen Spätsyndrome erfordern ein Armamentarium, und wir haben eher zu wenig als zu viele Optionen.

\section{Zählen nur noch Blockbuster?}

Bei den Medikamenten erleben wir eine Entwicklung, die wir aus vielen Bereichen kennen: An den Universitäten konzentrieren wir uns auf die großen Gebiete, Orchideenfächer werden immer seltener. Verlage bieten nur noch Bestseller an, seltene Themen werden nicht mehr bedient. Das trifft auch in der Neurologie zu, wo es dutzende Bücher zu den „wichtigen“ Krankheiten gibt und Neuropathien, Myopathien, autonomes Nervensystem etc. Stiefkinder sind, weil die zu erwartende Auflage zu gering ist.

\section{Die Reihen lichten sich}

In der Parkinson-Therapie mussten wir in den letzten Jahren bereits auf einige Substanzen verzichten. Das länger wirksame Levodopa-Präparat IPX066 (Levodopa plus Carbidopa) ist zugelassen, kommt in Deutschland aber nicht auf den Markt $\left(\right.$ Rytary $\left.^{\mathrm{mi}}\right)$. Die ergolinen Dopaminagonisten verschwinden langsam komplett. Tolcapon ist Mittel der zweiten Wahl und mit Auflagen (Leberwertkontrollen) belastet. Safinamid und Opicapon haben sehr gute Studienergebnisse und sind zugelassen, ihnen wurde aber vom G-BA kein Zusatznutzen attestiert, weshalb Safinamid beinahe vom Markt verschwunden wäre und die $\mathrm{Zu}$ kunft von Opicapon noch ungewiss ist. Dafür haben wir im Gegenzug unzählige generische Levodopa-Präparate und Dopaminagonisten, für die es keinerlei Studien gibt und deren Preis stellenweise über dem der Originale liegt (Rasagilin generisch ist teurer als Safinamid original, Entacapon und Tolcapon generisch sollen teurer sein als Opicapon original).

Auf der anderen Seite werden aktuell Medikamente zur Behandlung sehr seltener Krankheiten entwickelt, deren Preise extrem hoch sein dürfen, da sie sich unterhalb des Radars der Regularien befinden. Auch diese Medikamente könnten wieder verschwinden, wenn die Substanz generisch wird und der Ertrag den Aufwand unterschreitet.

Es trifft zu, dass ich nur wenige Patienten behandle, die gut von Budipin profitieren und für die es ein Verlust sein wird, wenn diese Substanz nicht mehr verfügbar ist. Aber es gibt viele neurologische Krankheiten, die ich noch seltener sehe sowie therapiere und bei denen ich mir ein wirksames Medikament wünschen würde.

Prof. Dr. med. Wolfgang Jost, Wolfach 